\title{
Antidepressant bupropion exerts alleviating properties in an ovariectomized osteoporotic rat model
}

\author{
Hatem M ABUOHASHISH ${ }^{1,2}$, Mohammed M AHMED ${ }^{1}$, Salim S AL-REJAIE ${ }^{1, *}$, Kamal EH ELTAHIR ${ }^{1}$ \\ ${ }^{1}$ Department of Pharmacology and Toxicology, College of Pharmacy, King Saud University, Riyadh 11544, Saudi Arabia; ${ }^{2}$ Department \\ of Biomedical Dental Sciences, College of Dentistry, Dammam University, Dammam 31441, Saudi Arabia
}

\begin{abstract}
Aim: Depression is a risk factor for impaired bone mass and micro-architecture, but several antidepressants were found to increase the incidence of osteoporotic fractures. In the present study we used ovariectomized (OVX) rats as a model of osteoporosis to investigate the effects of the antidepressant bupropion on the femoral bones.

Methods: OVX animals were treated with bupropion $\left(30,60 \mathrm{mg} \cdot \mathrm{kg}^{-1} \cdot \mathrm{d}^{-1}\right)$ for six weeks. Bone turnover biomarkers (urinary DPD/Cr ratio, serum BALP, OC, TRAcP 5b, CTX and SRANKL levels) and inflammatory cytokines (TNF- $\alpha$, IL-1 $\beta$ and IL-6) were determined using ELISA. Inductively coupled plasma mass spectroscopy (ICP-MS) was used to determine the femoral bone mineral concentrations. The cortical and trabecular morphometric parameters of femoral bones were determined using micro-CT scan and histopathology.

Results: In OVX rats, the levels of bone turnover biomarkers and inflammatory cytokines were significantly elevated and femoral bone $\mathrm{Ca}^{2+}$ and $\mathrm{PO}_{4}{ }^{3-}$ concentrations were significantly reduced. Moreover, cortical and trabecular morphometric parameters and histopathology of femoral bones were severely altered by ovariectomy. Bupropion dose-dependently inhibited the increases in bone turnover biomarkers and inflammatory cytokines. OVX rats treated with the high dose of bupropion showed normal mineral concentrations in femoral bones. The altered morphometric parameters and histopathology of femoral bones were markedly attenuated by the treatment.

Conclusion: Bupropion exerts osteo-protective action in OVX rats through suppressing osteoclastogenesis-inducing factors and inflammation, which stabilize the osteoclasts and decrease bone matrix degradation or resorption.
\end{abstract}

Keywords: antidepressant; bupropion; bone disease; osteoporosis; ovariectomized rat; inflammatory cytokines; micro-CT; osteoclastogenesis

Acta Pharmacologica Sinica (2015) 36: 209-220; doi: 10.1038/aps.2014.111; published online 29 Dec 2014

\section{Introduction}

Osteoporosis, a chronic disease of the skeleton in which bones become fragile due to a reduction in bone mass, is considered the most prevalent metabolic bone disease of humans ${ }^{[1,2]}$. Several epidemiological studies indicated that approximately 150 million people are suffering from osteoporosis worldwide ${ }^{[1-3]}$. The pathophysiological mechanisms of bone loss in osteoporosis include an imbalance between osteoclastic and osteoblastic function, where the osteoclastic bone resorption exceeds the osteoblastic bone formation ${ }^{[4]}$.

Numerous experimental models have been suggested to induce osteopenia and osteoporosis. Rats are the preferred experimental animal model for most researchers ${ }^{[5]}$. Among

\footnotetext{
* To whom correspondence should be addressed.

E-mail rejaie@ksu.edu.sa

Received 2014-05-02 Accepted 2014-08-20
}

these models, the rat OVX model is the best and most popular model to study bone loss in the proximal tibia, the distal femur, and the lumbar vertebrae. According to the FDA guidelines, the bone loss features in the rat OVX model imitate the bone changes following oophorectomy or menopause in human beings; thus this model is suitable for assessing the preventative potential of new therapeutic agents ${ }^{[6]}$. In addition to the OVX model, several other experimental models are used to induce bone loss. Rats are used to study the pathogenesis of decreased bone mass following alcohol abuse ${ }^{[7]}$. Surgical and conservative methods of immobilization are another method to induce osteoporosis ${ }^{[5]}$. A high cholesterol or lowcalcium diet was found to increase osteoporosis risk by attenuating osteoblast differentiation and proliferation ${ }^{[8,9]}$. Diabetic associated osteopenia has also been extensively investigated in several experimental studies ${ }^{[10,11]}$.

The linkage between depression and osteoporosis has been 
established in several clinical and epidemiological studies ${ }^{[12-14]}$. Indeed, depressed patients have a higher risk of developing osteoporotic fractures $^{[15]}$. Previous studies found that bone mass is generally low in patients with depression compared to non-depressed patients, especially within the total femur ${ }^{[16-19]}$. A population-based study demonstrated that elderly women aged $>50$ have a high risk of depression that associates with certain life changes, such as dissatisfaction, nervousness, loneliness, sleep disorders, uneasy feelings and a greater risk of hip fracture ${ }^{[20]}$. Physiological data that support the potential mechanisms of bone loss in depression are also present. Inflammatory cytokines play an important role in the etiology of both diseases. Inflammatory cytokines are known to be a strong inducer of osteoclastogenesis. The activity and level of pro-inflammatory cytokines, such as interleukin-1 $\beta$ (IL-1 $\beta$ ), interleukin-6 (IL-6) and tumor necrosis factor- $\alpha$ (TNF- $\alpha$ ), are also increased in depression ${ }^{[21]}$. Moreover, as potent stimulators of the hypothalamo-pituitary-adrenal (HPA) axis, these inflammatory mediators might contribute to hypercortisolism in depression ${ }^{[22]}$.

The use of antidepressants that have the potential to affect either bone strength or risk of fracture is a possible confounding factor in the association between depression and bone mineral density (BMD). In cross-sectional analyses, patients taking selective serotonin reuptake inhibitors (SSRIs), but not other classes of antidepressants, have experienced lower lumbar spine and hip BMD, particularly older patients ${ }^{[15,23,24]}$. In prospective analyses, current and baseline use of SSRI, but not tricyclic antidepressants (TCAs), have been associated with decreased hip BMD after 5 years of administration ${ }^{[25,26]}$. Most SSRIs associated with a dose-dependent increase in fracture risk and TCAs, such as amitriptyline and clomipramine, are also associated with the same level of fractures ${ }^{[22]}$. Animal studies also indicated that serotonin may influence bone mass. Battaglino et $a l^{[27]}$ demonstrated that mice injected daily with fluoxetine had increased bone formation. However, these effects are not observed in estrogen deficient animals; this finding suggests that the effect of antidepressants on bone metabolism may depend on sex steroids ${ }^{[28]}$.

Bupropion hydrochloride [( \pm )-2-tert-butylamino-3-chloropropiophenone $\mathrm{HCl}$ ] is an atypical antidepressant with stimulant properties. It is a nonselective inhibitor of the dopamine transporter (DAT) and the norepinephrine transporter (NET), and it is also an antagonist to neuronal nicotinic acetylcholine receptors (nAChRs) ${ }^{[29]}$. The neurochemical properties of bupropion are different from those of the commonly used TCAs; however, its antidepressant efficacy seems to be similar to that of SSRIs or TCAs ${ }^{[30]}$. Bupropion is also proposed to be an aid in smoking cessation ${ }^{[31]}$. Bupropion was found to inhibit inflammatory cytokine production in several studies. Clinical case reports have indicated that bupropion can reduce the activity of numerous diseases where inflammatory mediators are thought to be patho-physiologically active, such as atopic dermatitis and psoriasis ${ }^{[32]}$, Crohn's disease ${ }^{[33]}$, recurrent aphthous oral ulcers ${ }^{[34]}$, and hepatitis $\mathrm{B}^{[35]}$.

Large trials have demonstrated that bupropion induces remission of BMD loss in Crohn's disease and lowers TNF- $\mathrm{a}$ levels ${ }^{[33,36]}$. Brustolim and colleagues demonstrated that bupropion inhibited lipopolysaccharide (LPS)-induced inflammatory mediator release such as TNF- $\alpha$, interferon-gamma (IFN-gamma), IL-1 $\beta$, and nitric oxide (NO) ${ }^{[37]}$. Moreover, strong evidence exists that suggests that bupropion might be useful in inflammatory cytokine-mediated malignancies and diseases, such as chronic lymphocytic leukemia ${ }^{[35]}$, multiple myeloma $^{[38]}$, and osteoporosis. Thus, the present study was designed to investigate the potential protective properties of bupropion in an experimentally-induced model of osteoporosis in OVX Wistar rats.

\section{Materials and methods \\ Drugs and chemicals}

Bupropion $\mathrm{HCl}$ salt was purchased from Toronto Research Lab Co, Ltd (Toronto, Canada). Rat ELISA kits for deoxypyridinoline (DPD) cross links, bone specific alkaline phosphatase (BALP), osteocalcin (OC), tartrate resistant acid phosphatase (TRAcP), telopeptides of collagen type I (CTX) and soluble receptor activator of NF-KB ligand (sRANKL) were purchased from USCN LIFE, Wuhan EIAab Science Co, Ltd (Wuhan, China). Rat ELISA kits for tumor necrosis factor-a (TNF-a), interleukin $1 \beta$ (IL-1 $\beta$ ) and interleukin 6 (IL-6) were purchased from R\&D Systems (Minneapolis, USA).

\section{Experimental animals}

Forty female Wistar albino rats approximately three months of age were obtained from the Experimental Animal Care Center, College of Pharmacy, King Saud University, Riyadh, Saudi Arabia. All animals were maintained under controlled conditions of temperature $\left(22 \pm 1^{\circ} \mathrm{C}\right)$, humidity $(50 \%-55 \%)$ and light (12 h dark and $12 \mathrm{~h}$ light). The animals were provided free access to Purina rat chow (manufactured by Grain Silos and Flour Mills Organization, Riyadh, Saudi Arabia) and water ad libitum. The experimental procedures and protocols including euthanasia were in accordance with the National Institute of Health Guide for the Care and Use of Laboratory Animals, Institute for Laboratory Animal Research (NIH Publications № 80-23; 1996) as well as the Ethical Guidelines of the Experimental Animal Care Center, College of Pharmacy, King Saud University, Riyadh, Saudi Arabia.

\section{Induction of osteoporosis}

Osteoporosis was induced in thirty rats by bilateral ovariectomy as follows: under ether anesthesia, a longitudinal incision was made inferior to the rib cage on the dorsolateral body wall and the ovaries were exteriorized, ligated and excised ${ }^{[39]}$. The remaining ten rats were subjected to sham operation using the same procedure except for the ligation and excision steps. Topical antibiotic (fusidic acid) was administered twice weekly to eliminate the risk of postoperative infection.

\section{Experimental protocol}

Animals were allocated into four groups, ten animals each, as follows: (1) Sham, (2) OVX, (3) OVX+Bupropion (Bup) 30 
$\mathrm{mg} \cdot \mathrm{kg}^{-1} \cdot \mathrm{d}^{-1}$, and (4) OVX+Bupropion (Bup) $60 \mathrm{mg} \cdot \mathrm{kg}^{-1} \cdot \mathrm{d}^{-1}$. Doses for bupropion have been selected based on previous experimental reports ${ }^{[40,41]}$. One week after the ovariectomy and sham operations, treatment with bupropion was started. Bupropion $\mathrm{HCl}$ was dissolved in normal saline and given as an ip injection for 6 consecutive weeks. Sham and OVX control groups were treated with the vehicle ip during the treatments periods. The general health and behavior of the animals were monitored during the entire study. Body weights were recorded at $\mathrm{d} 0$ and then once a week for the duration of the study. Twenty-four hours after the last treatment, the rats were placed into metabolic cages and fasted for $16 \mathrm{~h}$. Afterwards, urine samples were collected and stored at $-20^{\circ} \mathrm{C}$ until further analysis. Under the deep ether anesthesia, blood samples were collected through cardiac puncture and then the animals were euthanized. The blood sample was left for $30 \mathrm{~min}$ at ambient temperature to coagulate and was then centrifuged at 4000 rounds per minute for $15 \mathrm{~min}$. Serum samples were separated and preserved at $-20^{\circ} \mathrm{C}$ until analyzed. The uterine tissues were removed, trimmed of fat, and weighed. Uterine tissue as a ratio of body weight $(\mathrm{g} / \mathrm{kg})$ was calculated. The right and left femoral bones of each rat were excised, cleaned to remove soft tissues, weighed and stored at $-20^{\circ} \mathrm{C}$ until analyzed.

\section{Estimation of bone metabolic biomarkers}

Urinary levels of deoxypyridinoline (DPD) cross links as well as serum levels of bone specific alkaline phosphatase (BALP), osteocalcin (OC), tartrate resistant acid phosphatase (TRAcP), telopeptides of collagen type I (CTX) and soluble receptor activator of NF-kB ligand (sRANKL) were estimated using rat ELISA kits (USCN LIFE, Wuhan EIAab Science Co, Ltd). Urinary creatinine levels were estimated using a commercially available kit (RANDOX Laboratories Ltd, Crumlin, UK). The urinary levels of DPD cross links are expressed as a ratio to creatinine.

\section{Estimation of inflammatory cytokines}

Serum levels of TNF- $\alpha$, IL-1 $\beta$, and IL- 6 were determined using commercially available rat ELISA kits (R\&D Systems, Minneapolis, USA).

\section{Estimation of femoral bone mineral concentrations}

One femoral bone from each rat was ashed in a muffle furnace (Lenton thermal designs, EF11/8 B) for $42 \mathrm{~h}$ at $650^{\circ} \mathrm{C}^{[1]}$. The ashed samples were weighed, and the ash/femoral bone ratio was calculated and expressed as a g/g ratio. From each sample, 0.1 gram of ash was then digested with $10 \mathrm{~mL}$ of $70 \%$ nitric acid. Samples were incubated overnight in a shaking water bath at $37^{\circ} \mathrm{C}$. The acidic ash mixture was diluted with deionized distilled water in a $1 / 9 \mathrm{ratio}^{[42]}$. The diluted samples were used to measure calcium $\left(\mathrm{Ca}^{2+}\right)$, inorganic phosphorus $\left(\mathrm{PO}_{4}{ }^{2-}\right)$ and magnesium $\left(\mathrm{Mg}^{2+}\right)$ concentrations. Inductively coupled plasma mass spectroscopy (ICP-MS, Perkin Elmer Sciex, ELAN DRC II, Shelton, USA) was used. Samples were decomposed to neutral elements in high temperature argon plasma and analyzed based on their mass to charge ratios. Mineral concentrations were measured in milligrams, and the results are expressed as milligrams of mineral to gram of ash.

\section{Micro-CT analysis}

Cortical and trabecular bone morphometric parameters were determined in the distal femoral bone using a high-resolution, cone-beam micro CT system (SkyScan 1172, SkyScan, Kontich, Belgium) that was kindly provided by Engineer Abdullah Bagshan Growth Factors Bone Regeneration Chair (GFBR), King Saud University, Riyadh, Saudi Arabia. The following parameters were measured: cortical thickness (Ct.Th), cortical crosssectional thickness (Ct.Cs.Th), cortical periosteal perimeter (Ct.Pe.Pm), cortical endosteal perimeter (Ct.En.Pm), cortical cross-sectional area (Ct.Ar), polar moment of inertia (MMI(p)), eccentricity (Ecc), cortical porosity (Ct.Po), trabecular bone mineral density (BMD), total volume (TV), bone volume (BV), percent bone volume (BV/TV), bone surface (BS), bone surface to volume ratio (BS/BV), bone surface density (BS/TV), trabecular thickness (Tb.Th), trabecular separation (Tb.Sp), trabecular number (Tb.N), trabecular pattern factor (Tb.Pf), structure model index (SMI), Euler connectivity (E.Con), Euler connectivity density (E.Con.D) and degree of anisotropy (DA). The bone samples were placed in a cylindrical holder, where the longitudinal axis of the bone and the sample holder were parallel to each other. Scans were performed using a $70 \mathrm{kV}$ applied voltage with a one mm aluminum filter. All cross sections contained $1024 \times 1240$ pixels with an isotropic voxel size of $10 \mu \mathrm{m}$. The data analysis was conducted using CT Analyzer 1.10.1.0 software (SkyScan, Kontich, Belgium). Depending on the length of the specimen, high resolution scanning was completed with slice number up to 1700 . All scanning conditions and reconstruction procedures were in accordance with manufacturer recommendations ${ }^{[10]}$.

\section{Femoral bone histopathology}

For bone histopathology, femur bones were fixed in 10\% neutral buffered formalin and stored in decal for $24 \mathrm{~h}$. Femur bones were then cut into several sections and embedded into paraffin wax blocks. Paraffin blocks were cut with a microtome and stained using hematoxylin and eosin (H\&E). The stained sections were mounted and observed for histopathological changes.

\section{Statistical analysis}

Statistical analyses were performed using Graph Pad Prism (version 5) software. All data are expressed as arithmetic mean values with their standard error of mean (mean \pm SEM). Statistical significance of differences was calculated using one-way analysis of variance (ANOVA) followed by post hoc Tukey-Kramer Multiple Comparison test or using two-way ANOVA followed by Bonferroni post hoc test. Statistical tests were used for comparisons between groups, and statistical significance was established at $P<0.05$. 


\section{Results}

\section{Effects on body and uterine weights}

The mean weights of all animals were between 250 to $270 \mathrm{~g}$ on the first day of the experiment. Four weeks after the ovariectomies, the rat body weights were markedly $(P<0.05)$ increased compared to the sham operated group; this difference became more significant at 5 and 6 weeks post operation. Bupropion treatment at either dose $\left(30\right.$ or $60 \mathrm{mg} \cdot \mathrm{kg}^{-1} \cdot \mathrm{d}^{-1}$ ) did not alter body weight significantly when compared to the untreated group. In contrast to the body weight increase, the uterine weights decreased $(P<0.01)$ in the OVX group when compared to the sham group of rats. However, bupropion treatment for 6 weeks did not affect the uterine weights of OVX rats compared to the untreated OVX group (Figure 1).

\section{Effects on bone metabolic biomarkers and inflammatory cytokines}

The urinary and serum levels of bone metabolic biomarkers were significantly altered in the OVX rats compared to the sham group (Figure 2). The urinary DPD/Cr ratio (Figure 2A) and serum levels of BALP, OC, TRAcP 5b, CTX, and sRANKL (Figure 2B-2F) were significantly $(P<0.01, P<0.01, P<0.01$, $P<0.05, P<0.01$, and $P<0.01$; respectively) increased in the OVX rats compared to the sham group. Bupropion $\left(60 \mathrm{mg} \cdot \mathrm{kg}^{-1} \cdot \mathrm{d}^{-1}\right)$ treatment for 6 weeks significantly $(P<0.05)$ lowered the elevated values of urinary $\mathrm{DPD} / \mathrm{Cr}$ ratio (Figure $2 \mathrm{~A}$ ) and serum OC, CTX, and sRANKL (Figure 2C, 2E, and 2F, respectively). The increased levels of serum BALP were inhibited markedly $(P<0.05)$ by both the bupropion $\left(30\right.$ and $\left.60 \mathrm{mg} \cdot \mathrm{kg}^{-1} \cdot \mathrm{d}^{-1}\right)$ doses after 6 weeks of treatment (Figure 2B).

The serum levels of pro-inflammatory cytokines TNF-a, IL-1 $\beta$, and IL-6 were significantly $(P<0.01, P<0.05$, and $P<0.05$;
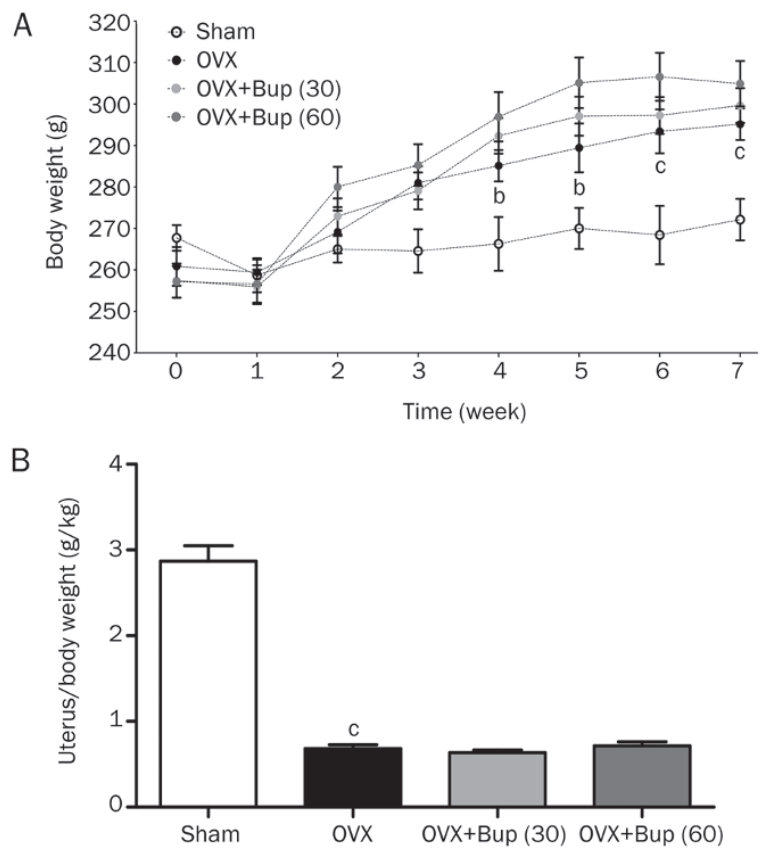

Figure 1. The effect of bupropion on the (A) body weights and (B) uterus/ body weight ratio of OVX rats. Data are expressed as the mean \pm SEM $(n=10)$ and analyzed using one-way ANOVA followed by post hoc TukeyKramer multiple comparisons test. ${ }^{\mathrm{b}} P<0.05,{ }^{\mathrm{c}} P<0.01$ compared with the sham group.

respectively) elevated in the OVX rats compared to the sham group, and these increased levels were significantly $(P<0.05)$ inhibited by only the higher dose $\left(60 \mathrm{mg} \cdot \mathrm{kg}^{-1} \cdot \mathrm{d}^{-1}\right.$, ip) of bupropion (Figure 3).
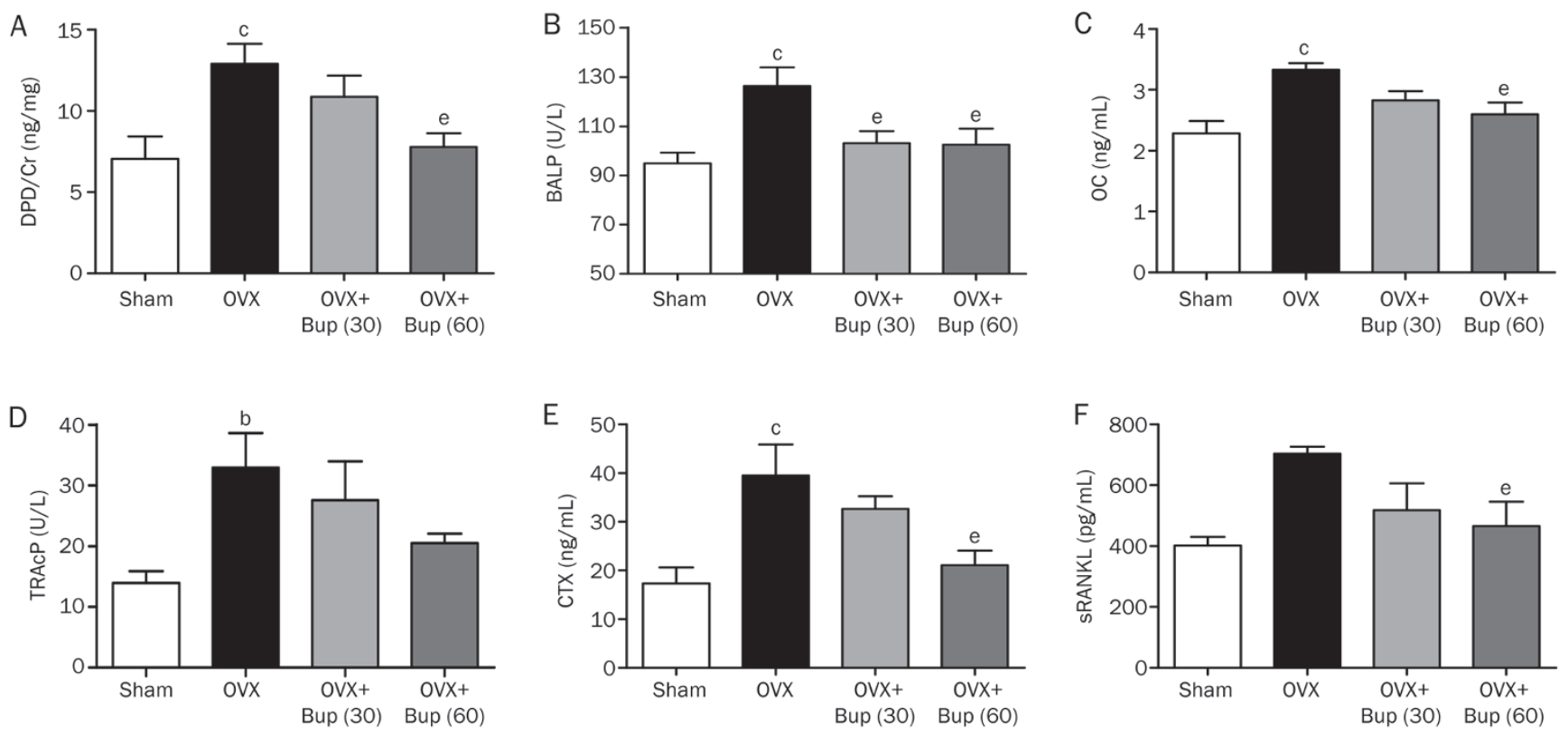

Figure 2. The effect of bupropion on the (A) urinary levels of DPD/Cr and serum levels of (B) BALP, (C) OC, (D) TRAP, (E) CTX, and (F) sRANKL in OVX rats. Data are expressed as the mean \pm SEM $(n=10)$ and analyzed using one-way ANOVA followed by post hoc Tukey-Kramer multiple comparisons test. ${ }^{\mathrm{b}} P<0.05,{ }^{\mathrm{c}} P<0.01$ compared with the sham group. ${ }^{\mathrm{e}} P<0.05$ compared with the OVX group. 

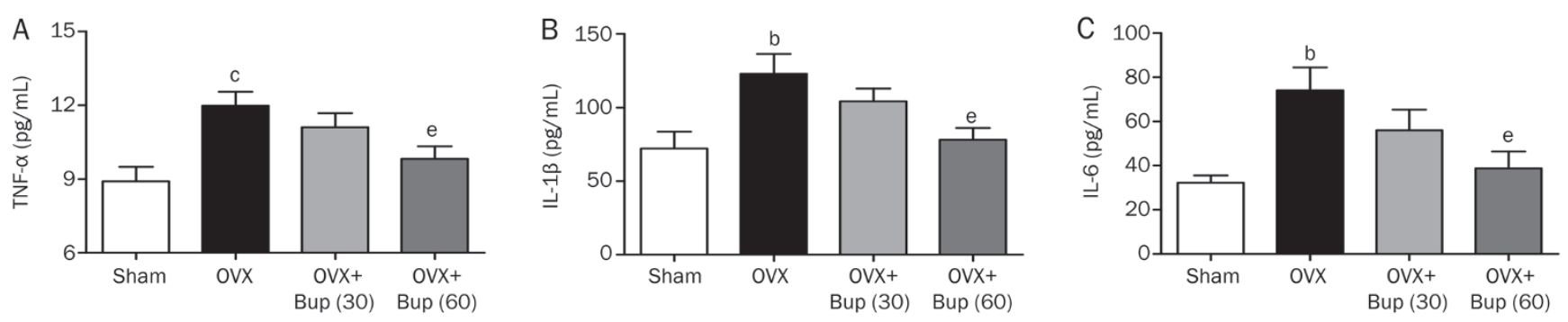

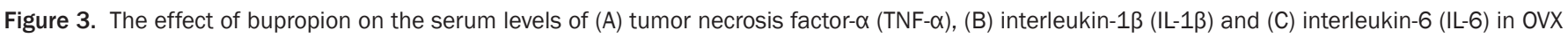
rats. Data are expressed as the mean \pm SEM $(n=10)$ and analyzed using one-way ANOVA followed by post hoc Tukey-Kramer multiple comparisons test. ${ }^{\mathrm{b}} P<0.05,{ }^{\mathrm{c}} P<0.01$ compared with the sham group. ${ }^{\mathrm{e}} P<0.05,{ }^{\mathrm{f}} P<0.01$ compared with the OVX group.

\section{Effects on femoral bone mineral concentrations}

Ash/bone weight ratio was significantly $(P<0.05)$ decreased in the OVX group compared to the sham group, and these values were increased $(P<0.05)$ after bupropion treatments of the OVX rats compared to the untreated OVX group. The concentrations of femoral bone minerals measured by ICPMS were highly affected by the OVX operation. The femoral bone $\mathrm{Ca}^{2+}$ and $\mathrm{PO}_{4}{ }^{2-}$ levels were significantly $(P<0.01)$ reduced in the OVX group compared to the sham group. Bupropion treatment with the higher dose $\left(60 \mathrm{mg} \cdot \mathrm{kg}^{-1} \cdot \mathrm{d}^{-1}\right)$ to the OVX rats for 6 weeks increased the bone levels of $\mathrm{Ca}^{2+}$ and $\mathrm{PO}_{4}{ }^{2-}$ significantly $(P<0.05)$ compared to the untreated OVX rats. However, the $\mathrm{Mg}^{2+}$ concentrations in femoral bones were not affected either by OVX operation or after the bupropion treatment to OVX rats (Table 1 ).

\section{Effects on bone morphometric parameters}

Micro CT scanning and analysis revealed that the rat femoral bones were severely affected by ovariectomy (Figure 4). The scanning results showed a general bone mass loss in the OVX group compared to the sham group. Micro CT scanning also confirmed that both doses of bupropion clearly induced remission of bone loss after 6 weeks in the OVX rats. Ovariectomy resulted in a significant alteration in cortical and trabecular morphometric parameters (Figures 5 and 6). Ct.Th, Ct.Cs.Th, Ct.Ar, MMI $(\mathrm{p})$ and Ecc were significantly $(P<0.01)$ reduced, while Ct.Pe.Pm, Ct.En.Pm and Ct.Po were increased in the untreated OVX animals (Figure 5). Bupropion administration to the OVX animals $\left(60 \mathrm{mg} \cdot \mathrm{kg}^{-1} \cdot \mathrm{d}^{-1}\right.$, ip) significantly $(P<0.01)$ inhibited both the decrease in the values of Ct.Th, Ct.Cs.Th, Ct.Ar, MMI(p) and Ecc $(P<0.05, P<0.05, P<0.05, P<0.01$, and $P<0.01$; respectively) and the increase in the values of $C t$.En. Pm and Ct.Po significantly $(P<0.05)$ (Figure 5). Moreover, there was a significant $(P<0.01)$ decrease in $\mathrm{BMD}, \mathrm{BV}, \mathrm{BV} / \mathrm{TV}$, BS, BS/TV, Tb.Th $(P<0.05)$, Tb.N, E.Con, E.Con.D and DA, while there was a significant $(P<0.01)$ increase in TV $(P<0.05)$, BS/BV, Tb.Sp, Tb.Pf and SMI compared to the sham control group (Figure 6). Except for SMI, 6 weeks of bupropion treatment at the higher dose $\left(60 \mathrm{mg} \cdot \mathrm{kg}^{-1} \cdot \mathrm{d}^{-1}\right.$, ip) significantly attenuated OVX-induced alterations in BMD $(P<0.01$; Figure $6 \mathrm{~A})$ and other trabecular morphometric parameters $(P<0.05$; Figure 6B-6O).

\section{Effects on bone histopathology}

Histopathological investigation of the rat femur bone sections revealed the following: (A) rats in the sham group expressed a well-formed and connected bone with normal thickness and density (Figure 7A); (B) femur bone tissues from the OVX group showed widely separated, uniform thinning of the trabeculae resulting in widened intertrabecular spaces with a moderate degree of osteoporosis (Figure 7B); (C) bupropion treatment at a dose of $30 \mathrm{mg} \cdot \mathrm{kg}^{-1} \cdot \mathrm{d}^{-1}$ resulted in a slight thinning of bone trabeculae and some degree of separation, revealing mild osteoporotic changes (Figure 7C); and (D) femur sections from the OVX+Bup $\left(60 \mathrm{mg} \cdot \mathrm{kg}^{-1} \cdot \mathrm{d}^{-1}\right)$ group demonstrated a well-formed, benign-looking joined bone trabeculae and cortex with a minimal degree of bone thinning and demineralization (Figure 7D).

Table 1. Effect of bupropion on ash/femoral bone weight ratio and femoral bone concentrations of $\mathrm{Ca}^{2+}, \mathrm{PO}_{4}{ }^{2-}$, and $\mathrm{Mg}^{2+}$ in female Wistar albino rats.

\begin{tabular}{lcccc}
\hline & $\begin{array}{c}\text { Ash/bone } \\
\text { weight ratio }\end{array}$ & $\begin{array}{c}\mathrm{Ca}^{2+} \\
\left(\mathrm{mg} \mathrm{Ca}{ }^{2+} / \mathrm{g} \text { ash }\right)\end{array}$ & $\begin{array}{c}\mathrm{PO}_{4}{ }^{2-} \\
\left(\mathrm{mg} \mathrm{PO}_{4}{ }^{2-} / \mathrm{g} \text { ash }\right)\end{array}$ & $\begin{array}{c}\mathrm{Mg}^{2+} \\
\left(\mathrm{mg} \mathrm{Mg}{ }^{2+} / \mathrm{g} \mathrm{ash}\right)\end{array}$ \\
\hline Sham & $0.576 \pm 0.006$ & $110.62 \pm 3.048$ & $103.37 \pm 2.581$ & $5.17 \pm 0.291$ \\
OVX & $0.522 \pm 0.012^{\mathrm{b}}$ & $91.09 \pm 5.475^{\mathrm{c}}$ & $87.05 \pm 4.743^{\mathrm{c}}$ & $4.79 \pm 0.281$ \\
OVX+Bup (30) & $0.572 \pm 0.013^{\mathrm{e}}$ & $96.58 \pm 2.237$ & $92.71 \pm 2.392$ & $4.94 \pm 0.381$ \\
OVX+Bup (60) & $0.569 \pm 0.014^{\mathrm{e}}$ & $106.39 \pm 3.253^{\mathrm{e}}$ & $101.09 \pm 2.8138^{\mathrm{e}}$ & $5.09 \pm 0.211$ \\
\hline
\end{tabular}

Data were expressed as mean \pm SEM and analyzed using one-way ANOVA followed by post hoc Tukey-Kramer multiple comparisons test ( $n=9 /$ group). ${ }^{\mathrm{b}} P<0.05,{ }^{\mathrm{c}} P<0.01$ compared with the sham group. ${ }^{\mathrm{e}} P<0.05$ compared with the OVX group. 

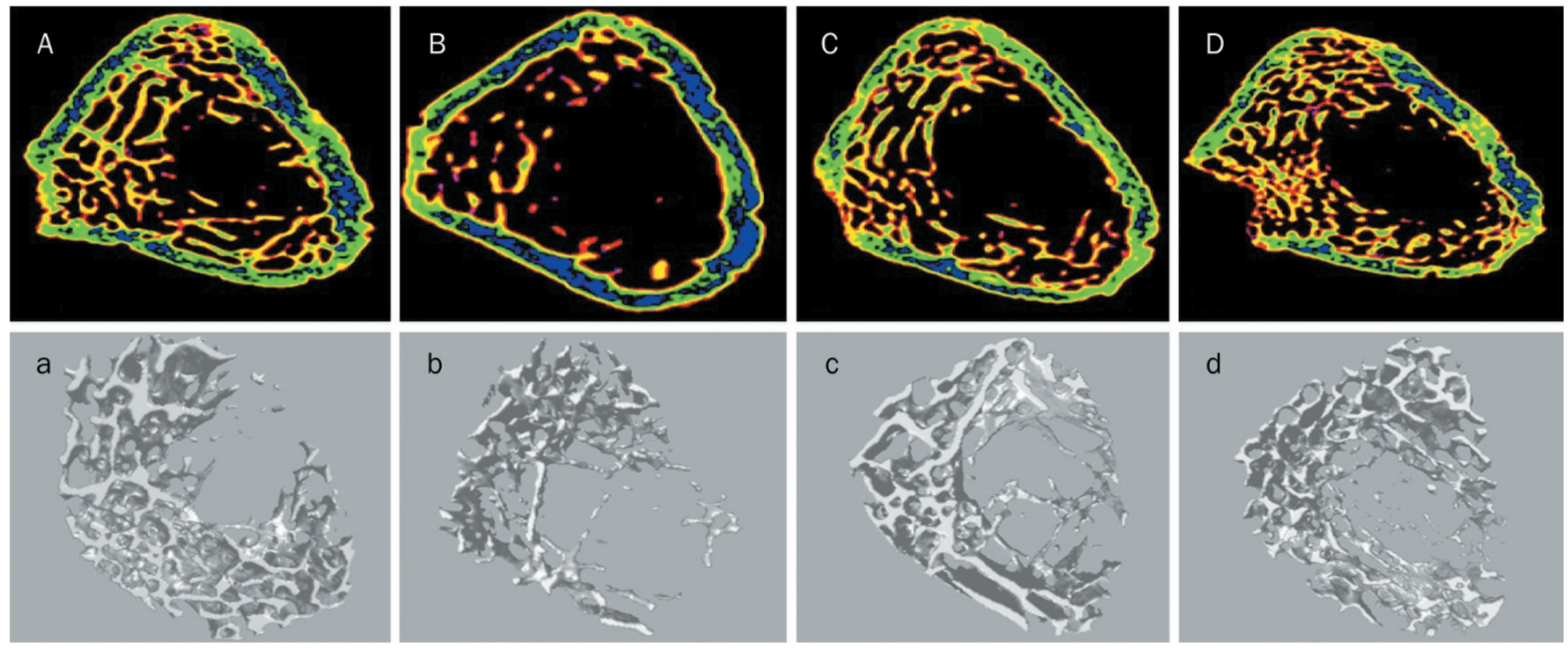

Figure 4. The effect of bupropion on trabecular bone micro-structure of OVX rats. Images (A-D) showed 2-dimensional transverse sections of the distal femoral bone. Images (a-d) showed 3-dimensional trabecular bone of the distal femoral bone. The sham group (A and a) is considered to exhibit wellformed bone mass with well-connected trabeculae. In the OVX rats, severe bone loss, thinning and separation of the trabecular bone was observed (B and b). The OVX+Bup (30) group exhibited a gradual restoration of bone mass and trabecular structure ( $\mathrm{C}$ and $\mathrm{C})$. The high dose of bupropion treatment to OVX rats exhibited a more prominent protective effect on bone mass and trabecular separation and thinning ( $D$ and $d)$.

\section{Discussion}

Bupropion is a well-known and clinically used antidepressant. To the best of our knowledge, bupropion was not investigated in an animal model of OVX-induced alterations and bone loss. Our results revealed that treatment of OVX rats with bupropion can correct the altered bone formation, resorption, reduced bone mineral levels and other bone biomarkers. The results also showed that bupropion induced remission in the altered cortical and trabecular morphometric parameters. Histopathological examinations further confirmed protective effects of bupropion treatment on OVX-induced changes in the rat femoral bones. Unlike bupropion, other antidepressants such as the well-known SSRIs (fluoxetine, sertraline and fluvoxamine) were found in earlier experimental studies to worsen bone metabolic biomarkers ${ }^{[33]}$ and mechanical properties $^{[4]}$ in OVX rats. In the current study, the effects of bupropion were not investigated with other antidepressants, such as SSRIs, as it is clinically used alone and not in combination with any other antidepressant. Earlier clinical studies conducted by Kast $e$ e $a l^{[36]}$ and Kane et $a l^{[33]}$ demonstrated that bupropion can ameliorate bone loss associated with Crohn's disease. Furthermore, the level of osteoclastogenesis inducing inflammatory cytokines was found to be reduced by bupropion ${ }^{[37,45]}$. This finding suggests that bupropion may be considered beneficial in managing the diseases where inflammation is thought to be patho-physiologically active, such as osteoporotic conditions.

In the present study, body weights of the OVX rats were significantly increased compared to the sham operated rats, which is in agreement with earlier reports ${ }^{[46-48]}$. It is well documented that estrogen deficiency in menopause is associated with a progressive increase in weight and redistribution of body fats. This effect is believed to be through interference with the normal action of leptin ${ }^{[49]}$. Estrogen administration was reported to reduce food intake and decrease body weight gain after menopause ${ }^{[50]}$, an effect that has been suggested to be regulated by estrogen receptor alpha (ERa). The lack of estrogen's control on food intake in the ER a-knockout mouse provides evidence for this regulation ${ }^{[51]}$. Estrogen is the main sex hormone produced in females by the ovaries ${ }^{[52]}$, which induces uterine growth. Present data revealed a significant decrease in uterine weight and an increase in body weight ratio in estrogen deficient animals, which is in agreement with earlier studies ${ }^{[39,46]}$. The decline in the uterine weight ensured the success of the ovariectomy ${ }^{[52]}$. Bupropion treatments to OVX rats did not attenuate the body weight gain, whereas the clinical data supported weight reduction in patients after bupropion use ${ }^{[53]}$. However, bupropion treatment for $15 \mathrm{~d}$ did not have a significant effect on body weight when compared with the control in an experimental study ${ }^{[54]}$. Therefore, further investigations are needed to compare the effect of bupropion on the body weight gain associated with menopause or other conditions. Additionally, administration of bupropion did not alter the decrease in uterine to body weight ratio in OVX rats; this finding suggests that bupropion does not have estrogenic activity and that the alleviating effects of bupropion on bone resorption do not depend on sex steroids.

Bone resorption metabolites are effective tools for the diagnosis of osteoporosis severity and development. DPD is a highly specific cross link of bone collagen, which is released into the circulation during bone resorption ${ }^{[55]}$. Because it is found predominantly in bone, it can be considered a sensitive marker for bone resorption and its urinary concentrations 

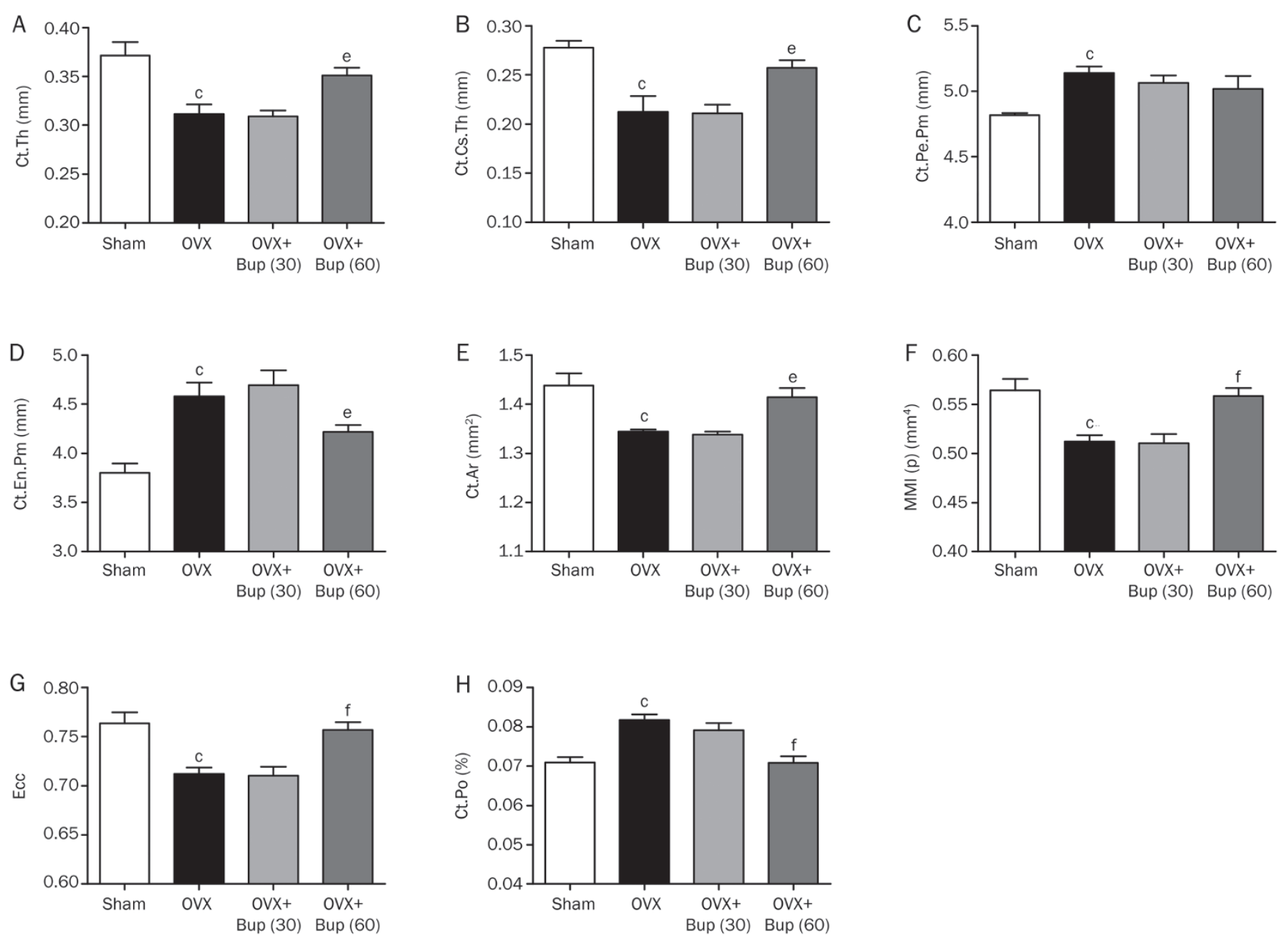

Figure 5. The effect of bupropion on cortical morphometric parameters measured by micro-CT in OVX rats. Data are expressed as the mean \pm SEM ( $n=10$ ) and analyzed using one-way ANOVA followed by post hoc Tukey-Kramer multiple comparisons test. ${ }^{\mathrm{c}} P<0.01$ compared with the sham group. ${ }^{\text {e }} P<0.05$, ${ }^{\mathrm{f}} P<0.01$ compared with the OVX group.

could therefore be an excellent marker for bone diseases, such as osteoporosis ${ }^{[56]}$. BALP is an enzyme that is synthesized by the osteoblasts and involved in the calcification of bone matrix ${ }^{[57]}$. Serum levels of BALP are known to be increased in conditions characterized by excessive bone turnover including postmenopausal osteoporosis ${ }^{[58]}$. OC plays a vital role in the bone mineralization process as it has a high affinity for calcium $^{[59]}$. Calcium and phosphorus deficiency during osteoporosis can lead to the elevation of free OC molecules in the circulation ${ }^{[59]}$. TRAcP is localized within the ruffled border area of the osteoclasts, where it plays a role in bone resorption via the production of destructive hydroxyl radicals ${ }^{[60,61]}$. CTX telopeptides are proteolytic fragments of type 1 collagen formed during bone resorption ${ }^{[62]}$. CTX telopeptides are sensitive and specific markers of bone resorption in the context of metabolic bone disease ${ }^{[63]}$. In the present work, ovariectomyinduced estrogen deficiency strongly activated the osteoclastic degradation of the bone matrix. Therefore, urinary and serum levels of the bone resorption biomarkers, such as DPD, BLAP, OC, TRACP, and CTX, were found to be significantly elevated in the OVX group compared to the sham operated group. In the current study, bupropion was found to alleviate the elevation of bone resorption biomarkers induced by ovariectomy. These alleviating effects seemed to be due to the inhibition of the osteoclastogenesis inducing cytokines, which stabilize the osteoclasts and reduce bone matrix degradation.

In the current investigation, the serum level of sRANKL, a potent osteoclast differentiation factor, was elevated in the OVX group compared to the sham group, which is in agreement with the results from previous studies ${ }^{[64,65]}$. RANKL is a membrane-bound secreted protein, which can be present as a membrane bound or insoluble (sRANKL) form ${ }^{[66]}$. Bone resorption is regulated by the interaction of RANK with its ligand, RANKL ${ }^{[67]}$. RANK is a type I homotrimeric transmembrane protein. It is a member of the tumor necrosis factor family expressed by osteoclast precursors, mature osteoclasts, dendritic cells and some cancer cells, such as breast and prostate cancers ${ }^{[6,67]}$. One possible explanation for the elevated sRANKL serum level is that bilateral ovariectomy could stimulate oxidative stress and the generation of reactive oxygen spe- 

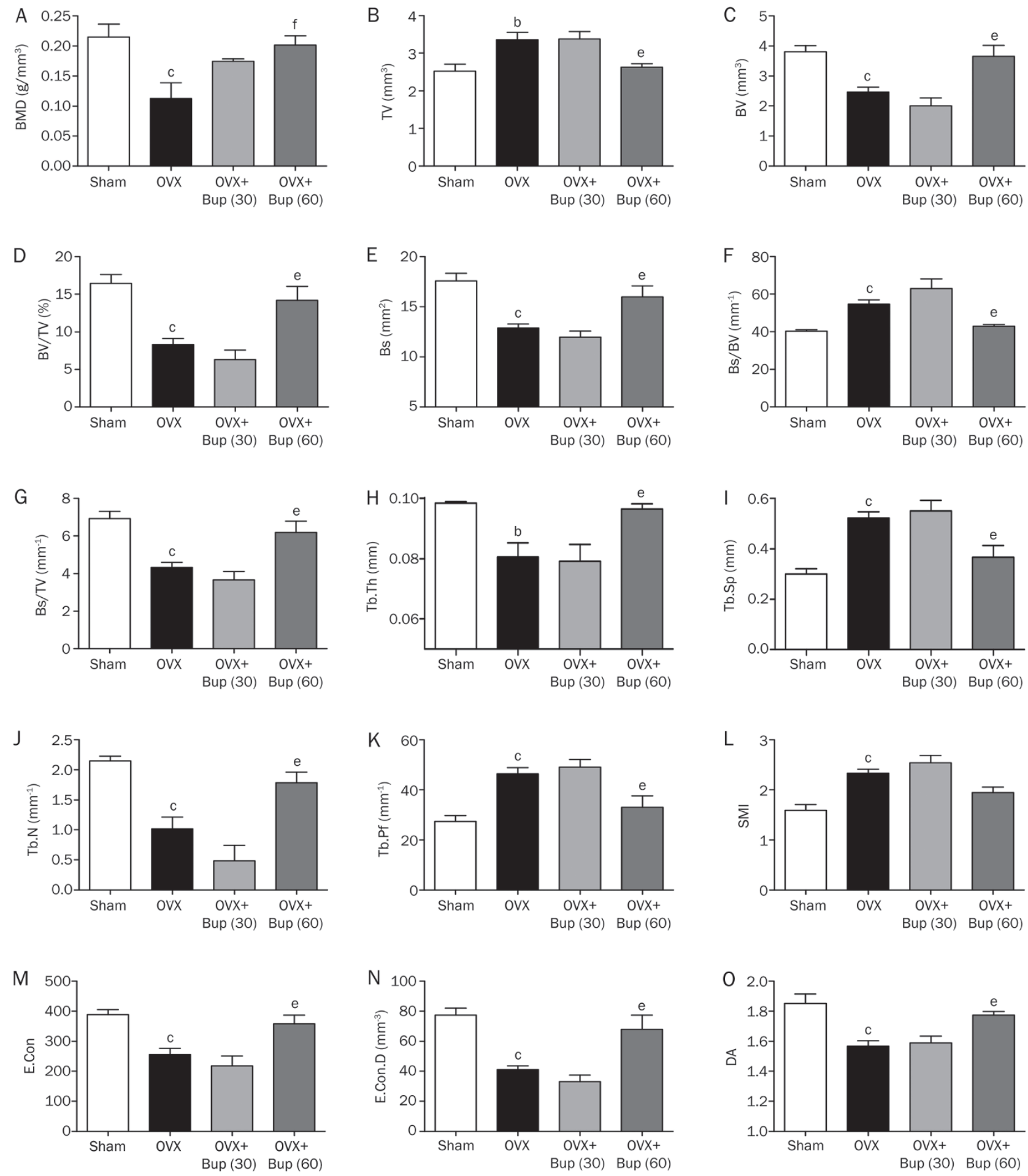

Figure 6. The effect of bupropion on trabecular morphometric parameters measured by micro-CT in OVX rats. Data are expressed as the mean \pm SEM $(n=10)$ and analyzed using one-way ANOVA followed by post hoc Tukey-Kramer multiple comparisons test. ${ }^{\mathrm{b}} P<0.05$, ${ }^{\mathrm{c}} P<0.01$ compared with the sham group. ${ }^{\mathrm{e}} \mathrm{P}<0.05$ compared with the OVX group.

cies (ROS). ROS can induce the production of inflammatory cytokines, which have been reported to induce sRANKL synthesis ${ }^{[68]}$. Moreover, TNF-a itself compared with RANKL has relatively weak osteoclastogenic activity but strongly enhances
RANKL-induced osteoclastogenesis additively ${ }^{[69]}$. We also suggest that these high levels of RANKL induce osteoclastic proliferation and activation, resulting in elevated levels of bone resorption biomarkers. The results of this study revealed 

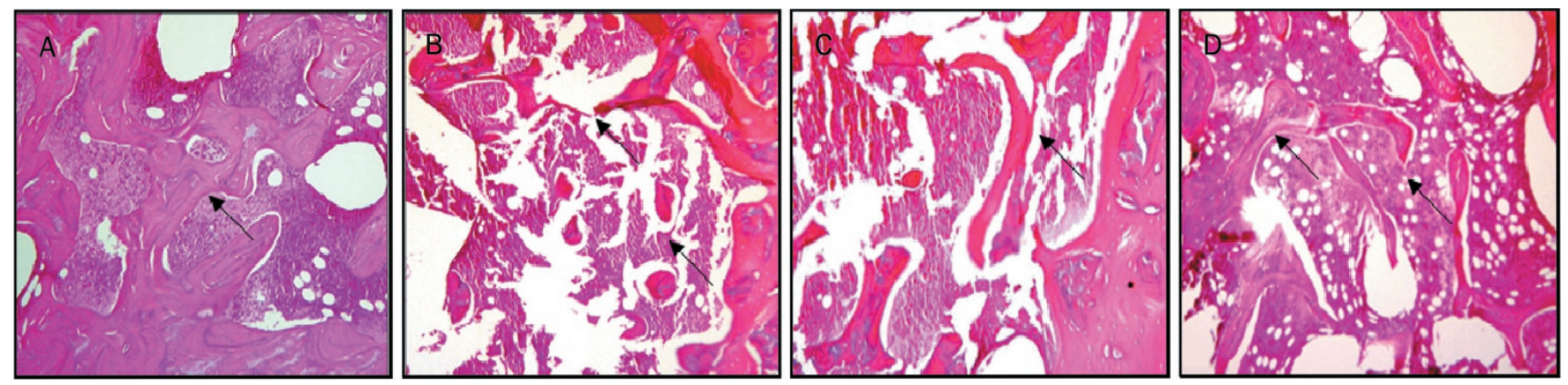

Figure 7. The effects of the ovariectomy and 6-week bupropion treatment (30 and $60 \mathrm{mg} \cdot \mathrm{kg}^{-1} \cdot \mathrm{d}^{-1}$, ip) on histopathological sections of female Wistar albino rat distal femoral bones, which exhibit the following: (A) well-formed, normal, connected and mature bony trabecula with a benign bone density and bone thickness. The histopathological analysis reports normal bone; (B) widely separated, thin-walled trabecular bone containing bone marrow element. The histopathological analysis reveals a moderate degree of osteoporosis; (C) disconnected, thin-walled trabecular bone along with a mild degree of trabecular bone separation. The histopathological analysis reveals a mild degree of osteoporosis; (D) minor degree thinning and separation of trabecula containing bone marrow element. The histopathological analysis reveals a minor degree of osteoporosis.

that bupropion administration attenuated the elevated levels of serum sRANKL; this effect could be mediated through bupropion's ability to reduce inflammatory cytokines activity, which is reported in the current and previous studies ${ }^{[35,37,45]}$.

In the present study, serum levels of bone resorbing inflammatory cytokines were significantly elevated in the OVX group. These findings are in agreement with earlier reports $^{[64,70]}$. Inflammatory cytokines such as TNF-a, IL-1 $\beta$, and IL-6 activate and increase the number of circulating osteoclast precursors, which can promote the proliferation of these cells to mature osteoclasts. This can activate the osteoclasts along with the release of more inflammatory mediators ${ }^{[66]}$. TNF- $\alpha$, IL-1 $\beta$, and IL- 6 can induce osteoclast differentiation and activation through tumor necrosis factor receptor activating factors (TRAF), mainly type 2 and $6^{[71]}$. The rise of inflammatory cytokine serum levels might also occur because of the oxidative stress induced by the ovariectomy. Bilateral ovariectomy and the resulting estrogen deficiency can enhance the development of oxidative stress and ROS. ROS are known potent NF-kB stimuli that mediate the expression of pro-inflammatory cytokines from different types of cells, including mononuclear cells ${ }^{[64,72]}$. In the present study, bupropion treatment resulted in a reduction in serum levels of these inflammatory mediators, which is consistent with other studies $^{[35,37,45]}$. Bupropion is believed to downregulate the synthesis of TNF-a, which could reduce TNF-a signaling without shifting the TNFR1/TNFR2 balance ${ }^{[45]}$.

The ovariectomy-induced decrease in ash/bone weight ratio and mineral concentrations were generally attenuated by bupropion treatment of the OVX rats. The ash/bone weight ratio and bone $\mathrm{Ca}^{2+}$ and $\mathrm{PO}_{4}{ }^{2-}$ were increased in bupropion treated animals, especially at the higher doses. However, bone $\mathrm{Mg}^{2+}$ concentrations were not significantly altered by ovariectomy with or without the antidepressant. The mechanism underlying the ash/bone weight ratio and bone mineral concentration decrease in the OVX group is the increased osteoclastic bone resorping activity due to the increased level of osteoclastogenesis-inducing cytokines. As mentioned earlier, osteoclast activity in bone resorption and demineralization are controlled by several factors including RANKL and inflammatory cytokines. In this study, these osteoclastogenesis factors are elevated in the OVX group. We suggest that the bone demineralization-alleviating effects of bupropion are due to its ability to reduce the levels of osteoclastogenesis-inducing cytokines, as reported here. As a result of decreased levels of these cytokines, osteoclast demineralization properties were stabilized, leading to an increase in ash/bone weight ratio and bone mineral concentration.

Micro CT scanning and analysis showed that cortical and trabecular bone morphometric parameters were also markedly altered by ovariectomy in the absence of bupropion treatments. Ct.Th, Ct.Cs.Th, Ct.Ar, MMI(p), Ecc, BMD, BV, BV/TV, BS, BS/TV, Tb.Th, Tb.N, E.Con, E.Con.D and DA were severely decreased, while Ct.Pe.Pm, Ct.En.Pm, Ct.Po, TV, BS/BV, Tb.Sp, Tb.Pf and SMI were significantly increased in the OVX group compared to the sham group. These alterations provide evidence for increased osteoclastic activity due to estrogen deficiency ${ }^{[73]}$. It is known that estrogen can inhibit bone resorption and destruction by controlling the number and the activity of osteoclasts ${ }^{[74]}$. Estrogen controls FasL gene expression in osteoclasts via ERa, which induces osteoclast apoptosis and decreases the life span these bone resorping cells ${ }^{[74]}$. The results of the current study also showed that treatment with bupropion, especially the higher dose (60 $\mathrm{mg} \cdot \mathrm{kg}^{-1} \cdot \mathrm{d}^{-1}$ ) for 6 consecutive weeks, in the OVX rats can significantly attenuate the altered cortical and trabecular morphometric parameters compared to the OVX control animals. These finding are consistent with other studies, where bupropion was reported to ameliorate Crohn's disease associated bone density reduction ${ }^{[3,36]}$ through its ability to lower inflammatory mediators ${ }^{[45]}$, an effect also reported here.

The results of the histopathological examination added new evidence that bilateral ovariectomy can affect bone mass. Obviously, estrogen deficiency resulted in bone resorption 
and destruction. Animals in the OVX group exhibited bone mass loss, especially in the trabecular bones. The femoral bones in the OVX group showed a widely separated and thin walled trabecular bone. Similar findings were observed in different studies ${ }^{[48,74]}$. However, histopathological examination of the femoral bones of bupropion-treated animals revealed a marked restorative action, which seems to be due to an increase in bone formation along with a reduction in bone resorption and destruction. These finding are in agreement with the biochemical and micro CT screening outcomes.

In conclusion, while bupropion did not have estrogenic activity, it did show osteo-protective effects on an ovariectomy-induced model of osteoporosis in Wistar rats. A possible mechanism of these osteo-protective effects is the ability of the antidepressant to inhibit the levels of osteoclastogenesisinducing factors such as RANKL and inflammatory mediators elevated by estrogen deficiency, which stabilize the stimulated osteoclasts and prevent the induction of bone resorption and destruction. In the present study, two doses of bupropion (30 and $60 \mathrm{mg} / \mathrm{kg}$ ) were administered. According to FDA guidelines, animal doses can be transformed to human-equivalent doses (HED) ${ }^{[75,76]}$. Therefore, the doses of bupropion used in this study can be transformed to human doses (4.8 and $9.2 \mathrm{mg} / \mathrm{kg}$ ). The maximum utilized daily dose of bupropion for the treatment of major depressive disorder is $450 \mathrm{mg}(\approx 6$ $\mathrm{mg} / \mathrm{kg})^{[2,77]}$, which indicates that the osteo-protective effects of bupropion could be achieved within the therapeutic range.

\section{Acknowledgements}

The authors would like to extend their deep appreciation to the following: (1) King Abdulaziz City for Science and Technology (KACST) for funding this research work via project № (LGP-32-33), (2) Engineer Abdullah Bagshan for Growth Factors and Bone Regeneration Chair (GFBR) at King Saud University for their kind support and providing micro $\mathrm{CT}$ and (3) Experimental Animal Care Center, College of Pharmacy, King Saud University for the technical support.

\section{Author contribution}

Hatem M ABUOHASHISH, Salim S AL-REJAIE, and Kamal EH ELTAHIR contributed to the study design; Hatem $M$ ABUOHASHISH and Mohammed M AHMED performed the research, conducted the data analysis, and wrote the manuscript.

\section{References}

1 Grases F, Sanchis P, Prieto RM, Perello J, Lopez-Gonzalez AA. Effect of tetracalcium dimagnesium phytate on bone characteristics in ovariectomized rats. J Med Food 2010; 13: 1301-6.

2 Potu BK, Rao MS, Nampurath GK, Chamallamudi MR, Prasad K, Nayak SR, et al. Evidence-based assessment of antiosteoporotic activity of petroleum-ether extract of Cissus quadrangularis Linn. on ovariectomy-induced osteoporosis. Ups J Med Sci 2009; 114: 140-8.

3 Khosla S, Westendorf JJ, Oursler MJ. Building bone to reverse osteoporosis and repair fractures. J Clin Invest 2008; 118: 421-8.

4 Riggs BL, Khosla S, Melton LJ 3rd. Sex steroids and the construction and conservation of the adult skeleton. Endocr Rev 2002; 23: 279-
302.

5 Lelovas PP, Xanthos TT, Thoma SE, Lyritis GP, Dontas IA. The laboratory rat as an animal model for osteoporosis research. Comp Med 2008; 58: 424-30.

6 Thompson DD, Simmons HA, Pirie CM, Ke HZ. FDA Guidelines and animal models for osteoporosis. Bone 1995; 17: 125S-133S.

7 Abukhadir SS, Mohamed N. Pathogenesis of alcohol-induced osteoporosis and its treatment: a review. Curr Drug Targets 2013; 14 : 1601-10.

8 You L, Sheng ZY, Tang CL, Chen L, Pan L, Chen JY. High cholesterol diet increases osteoporosis risk via inhibiting bone formation in rats. Acta Pharmacol Sin 2011; 32: 1498-504.

9 Seto H, Aoki K, Kasugai S, Ohya K. Trabecular bone turnover, bone marrow cell development, and gene expression of bone matrix proteins after low calcium feeding in rats. Bone 1999; 25: 687-95.

10 Abuohashish HM, Al-Rejaie SS, Al-Hosaini KA, Parmar MY, Ahmed MM. Alleviating effects of morin against experimentally-induced diabetic osteopenia. Diabetol Metab Syndr 2013; 5: 5.

11 Li B, Wang Y, Liu Y, Ma J, Li Y. Altered gene expression involved in insulin signaling pathway in type II diabetic osteoporosis rats model. Endocrine 2013; 43: 136-46.

12 Schweiger U, Deuschle M, Korner A, Lammers CH, Schmider J, Gotthardt U, et al. Low lumbar bone mineral density in patients with major depression. Am J Psychiatry 1994; 151: 1691-3.

13 Halbreich U, Rojansky N, Palter S, Hreshchyshyn M, Kreeger J, Bakhai $\mathrm{Y}$, et al. Decreased bone mineral density in medicated psychiatric patients. Psychosom Med 1995; 57: 485-91.

14 Michelson D, Stratakis C, Hill L, Reynolds J, Galliven E, Chrousos G, et al. Bone mineral density in women with depression. N Engl J Med 1996; 335: 1176-81.

15 Whooley MA, Kip KE, Cauley JA, Ensrud KE, Nevitt MC, Browner WS. Depression, falls, and risk of fracture in older women. Study of Osteoporotic Fractures Research Group. Arch Intern Med 1999; 159: 484-90.

16 Esel E, Ozsoy S, Tutus A, Sofuoglu S, Kartalci S, Bayram F, et al. Effects of antidepressant treatment and of gender on serum leptin levels in patients with major depression. Prog Neuropsychopharmacol Biol Psychiatry 2005; 29: 565-70.

17 Mussolino ME, Jonas BS, Looker AC. Depression and bone mineral density in young adults: results from NHANES III. Psychosom Med 2004; 66: 533-7.

18 Whooley MA, Cauley JA, Zmuda JM, Haney EM, Glynn NW. Depressive symptoms and bone mineral density in older men. J Geriatr Psychiatry Neurol 2004; 17: 88-92.

19 Wong SY, Lau EM, Lynn H, Leung PC, Woo J, Cummings SR, et al. Depression and bone mineral density: is there a relationship in elderly Asian men? Results from Mr Os (Hong Kong). Osteoporos Int 2005; 16: $610-5$.

20 Forsen L, Meyer HE, Sogaard AJ, Naess S, Schei B, Edna TH. Mental distress and risk of hip fracture. Do broken hearts lead to broken bones? J Epidemiol Community Health 1999; 53: 343-7.

21 Marques-Deak A, Cizza G, Sternberg E. Brain-immune interactions and disease susceptibility. Mol Psychiatry 2005; 10: 239-50.

22 Cizza G, Primma S, Csako G. Depression as a risk factor for osteoporosis. Trends Endocrinol Metab 2009; 20: 367-73.

23 Charney DS, Manji HK. Life stress, genes, and depression: multiple pathways lead to increased risk and new opportunities for intervention. Sci STKE 2004; 2004: re5.

24 Patterson-Buckendahl PE, Grindeland RE, Shakes DC, Morey-Holton ER, Cann CE. Circulating osteocalcin in rats is inversely responsive to changes in corticosterone. Am J Physiol 1988; 254: R828-33. 
25 Patterson-Buckendahl P, Kvetnansky R, Fukuhara K, Cizza G, Cann C. Regulation of plasma osteocalcin by corticosterone and norepinephrine during restraint stress. Bone 1995; 17: 467-72.

26 Patterson-Buckendahl P, Rusnak M, Fukuhara K, Kvetnansky R. Repeated immobilization stress reduces rat vertebral bone growth and osteocalcin. Am J Physiol Regul Integr Comp Physiol 2001; 280: R79-86.

27 Battaglino R, Fu J, Spate U, Ersoy U, Joe M, Sedaghat L, et al. Serotonin regulates osteoclast differentiation through its transporter. J Bone Miner Res 2004; 19: 1420-31.

28 Kendler KS, Karkowski LM, Prescott CA. Causal relationship between stressful life events and the onset of major depression. Am J Psychiatry 1999; 156: 837-41.

29 Dwoskin LP, Rauhut AS, King-Pospisil KA, Bardo MT. Review of the pharmacology and clinical profile of bupropion, an antidepressant and tobacco use cessation agent. CNS Drug Rev 2006; 12: 178-207.

30 Bredeloux P, Dubuc I, Costentin J. Comparisons between bupropion and dexamphetamine in a range of in vivo tests exploring dopaminergic transmission. Br J Pharmacol 2007; 150: 711-9.

31 Hurt RD, Sachs DP, Glover ED, Offord KP, Johnston JA, Dale LC, et al. A comparison of sustained-release bupropion and placebo for smoking cessation. N Engl J Med 1997; 337: 1195-202.

32 Altschuler EL, Kast RE. Bupropion in psoriasis and atopic dermatitis: decreased tumor necrosis factor-alpha? Psychosom Med 2003; 65: 719 .

33 Kane S, Altschuler EL, Kast RE. Crohn's disease remission on bupropion. Gastroenterology 2003; 125: 1290.

34 Kast RE, Altschuler EL. Bupropion and chronic aphthous ulceration. Arch Dermatol 2005; 141: 1167.

35 Kast RE. Anti- and pro-inflammatory considerations in antidepressant use during medical illness: bupropion lowers and mirtazapine increases circulating tumor necrosis factor-alpha levels. Gen Hosp Psychiatry 2003; 25: 495-6.

36 Kast RE, Altschuler EL. Remission of Crohn's disease on bupropion. Gastroenterology 2001; 121: 1260-1.

37 Brustolim D, Ribeiro-dos-Santos R, Kast RE, Altschuler EL, Soares MB. A new chapter opens in anti-inflammatory treatments: the antidepressant bupropion lowers production of tumor necrosis factoralpha and interferon-gamma in mice. Int Immunopharmacol 2006; 6: 903-7.

38 Kast RE. Evidence of a mechanism by which etanercept increased TNF-alpha in multiple myeloma: new insights into the biology of TNFalpha giving new treatment opportunities - the role of bupropion. Leuk Res 2005; 29: 1459-63.

39 Kaczmarczyk-Sedlak I, Folwarczna J, Trzeciak HI. Thalidomide affects the skeletal system of ovariectomized rats. Pharmacol Rep 2009; 61: 529-38.

40 Lee HJ, Rao JS, Chang L, Rapoport SI, Kim HW. Chronic imipramine but not bupropion increases arachidonic acid signaling in rat brain: is this related to 'switching' in bipolar disorder? Mol Psychiatry 2010; 15: 602-14.

41 Reichel CM, Murray JE, Grant KM, Bevins RA. Bupropion attenuates methamphetamine self-administration in adult male rats. Drug Alcohol Depend 2009; 100: 54-62.

42 Shahnazari M, Martin BR, Legette LL, Lachcik PJ, Welch J, Weaver CM. Diet calcium level but not calcium supplement particle size affects bone density and mechanical properties in ovariectomized rats. J Nutr 2009; 139: 1308-14.

43 El-Moursy SA, Abdalla AY, El-Ashmony SM, Rashed L, Ali HHM. The effect of two selective serotonin reuptake inhibitors on the bone turnover of intact and ovariectomized female albino rats. Res J Med
Med Sci 2009; 4: 136-50.

44 Folwarczna J, Pytlik M, Nowinska B, Cegiela U, Molin P, Hanke T, et al. Effects of selective serotonin reuptake inhibitors on bone mechanical properties in ovariectomized and non-ovariectomized rats. Bone 2009; 44: S339-S450.

45 Kast RE, Altschuler EL. Anti-apoptosis function of TNF-alpha in chronic lymphocytic leukemia: lessons from Crohn's disease and the therapeutic potential of bupropion to lower TNF-alpha. Arch Immunol Ther Exp (Warsz) 2005; 53: 143-7.

46 Hertrampf T, Schleipen B, Offermanns C, Velders M, Laudenbach U, Diel $P$. Comparison of the bone protective effects of an isoflavone-rich diet with dietary and subcutaneous administrations of genistein in ovariectomized rats. Toxicol Lett 2009; 184: 198-203.

47 Choi MJ. Effects of taurine supplementation on bone mineral density in ovariectomized rats fed calcium deficient diet. Nutr Res Pract 2009; 3: 108-13.

$48 \mathrm{Nian} \mathrm{H}$, Ma MH, Nian SS, Xu LL. Antiosteoporotic activity of icariin in ovariectomized rats. Phytomedicine 2009; 16: 320-6.

49 Gao Q, Horvath TL. Cross-talk between estrogen and leptin signaling in the hypothalamus. Am J Physiol Endocrinol Metab 2008; 294: E817-26.

50 Brown NM, Belles CA, Lindley SL, Zimmer-Nechemias LD, Zhao X, Witte DP, et al. The chemopreventive action of equol enantiomers in a chemically induced animal model of breast cancer. Carcinogenesis 2010; 31: 886-93.

51 Geary N, Asarian L, Korach KS, Pfaff DW, Ogawa S. Deficits in E2dependent control of feeding, weight gain, and cholecystokinin satiation in ER-alpha null mice. Endocrinology 2001; 142: 4751-7.

52 Kim MK, Kim HD, Park JH, Lim JI, Yang JS, Kwak WY, et al. An orally active cathepsin $\mathrm{K}$ inhibitor, furan-2-carboxylic acid, 1-\{1-[4-fluoro-2-(2oxo-pyrrolidin-1-yl)-phenyl]-3-oxo-piperidin-4-ylcarbamoyl\}-c yclohexyl)amide (OST-4077), inhibits osteoclast activity in vitro and bone loss in ovariectomized rats. J Pharmacol Exp Ther 2006; 318: 555-62.

53 Gadde KM, Parker CB, Maner LG, Wagner HR 2nd, Logue EJ, Drezner $\mathrm{MK}$, et al. Bupropion for weight loss: an investigation of efficacy and tolerability in overweight and obese women. Obes Res 2001; 9: 54451.

54 Santamaria A, Arias HR. Neurochemical and behavioral effects elicited by bupropion and diethylpropion in rats. Behav Brain Res 2010; 211: 132-9.

55 Hou MF, Lin SB, Yuan SS, Tsai LY, Tsai SM, Hsieh JS, et al. Diagnostic value of urine deoxypyridinoline for detecting bone metastases in breast cancer patients. Ann Clin Lab Sci 2003; 33: 55-61.

56 Samma S, Kagebayashi Y, Yasukawa M, Fukui Y, Ozono S, Hirao Y, et al. Sequential changes of urinary pyridinoline and deoxypyridinoline as markers of metastatic bone tumor in patients with prostate cancer: a preliminary study. Jpn J Clin Oncol 1997; 27: 26-30.

57 Sara F, Saygılı F. Causes of high bone alkaline phosphatase. Biotechnol Biotec Eq 2007; 21: 194-97.

58 Ross PD, Knowlton W. Rapid bone loss is associated with increased levels of biochemical markers. J Bone Miner Res 1998; 13: 297 302.

59 Jagtap VR, Ganu JV, Nagane NS. BMD and serum intact osteocalcin in postmenopausal osteoporosis women. Indian J Clin Biochem 2011; 26: 70-3.

60 Ljusberg J, Wang Y, Lang P, Norgard M, Dodds R, Hultenby K, et al. Proteolytic excision of a repressive loop domain in tartrate-resistant acid phosphatase by cathepsin K in osteoclasts. J Biol Chem 2005; 280: $28370-81$.

61 Lee H, Alam M, Seol J, Kim N. Tartrate-resistant acid phosphatase, matrix metalloproteinase-2 and tissue inhibitor of metalloproteinase-2 
in early stages of canine osteoarthritis. Veterinarni Medicina 2008; 53: $214-20$

62 Vasikaran SD. Utility of biochemical markers of bone turnover and bone mineral density in management of osteoporosis. Crit Rev Clin Lab Sci 2008; 45: 221-58.

63 Kurban S, Mehmetoglu I, Gönen S, Akbulut S. Investigation of serum crosslinked $\mathrm{N}$-telopeptides of type I collagen (NTx) levels and total antioxidant capacity in patients with type 2 diabetes mellitus. Turk J Biochem 2010; 35: 145-50.

64 Das AS, Mukherjee M, Das D, Mitra C. Protective action of aqueous black tea (Camellia sinensis) extract (BTE) against ovariectomyinduced oxidative stress of mononuclear cells and its associated progression of bone loss. Phytother Res 2009; 23: 1287-94.

65 Ominsky MS, Li X, Asuncion FJ, Barrero M, Warmington KS, Dwyer $D$, et al. RANKL inhibition with osteoprotegerin increases bone strength by improving cortical and trabecular bone architecture in ovariectomized rats. J Bone Miner Res 2008; 23: 672-82.

66 Boyce BF, Xing L. Biology of RANK, RANKL, and osteoprotegerin. Arthritis Res Ther 2007; 9: S1.

67 Deal C. Potential new drug targets for osteoporosis. Nat Clin Pract Rheumatol 2009; 5: 20-7.

68 Wei S, Kitaura H, Zhou P, Ross FP, Teitelbaum SL. IL-1 mediates TNFinduced osteoclastogenesis. J Clin Invest 2005; 115: 282-90.

69 Sato T, Watanabe K, Masuhara M, Hada N, Hakeda Y. Production of IL-7 is increased in ovariectomized mice, but not RANKL mRNA expression by osteoblasts/stromal cells in bone, and IL-7 enhances generation of osteoclast precursors in vitro. J Bone Miner Metab 2007; 25: 19-27.

70 Li B, Yu S. Genistein prevents bone resorption diseases by inhibiting bone resorption and stimulating bone formation. Biol Pharm Bull 2003; 26: 780-6.

71 Udagawa N, Kotake S, Kamatani N, Takahashi N, Suda T. The molecular mechanism of osteoclastogenesis in rheumatoid arthritis. Arthritis Res 2002; 4: 281-9.

72 Pfeilschifter J, Koditz R, Pfohl M, Schatz H. Changes in proinflammatory cytokine activity after menopause. Endocr Rev 2002; 23: 90-119.

73 Kennedy OD, Brennan O, Rackard SM, Staines A, O'Brien FJ, Taylor D, et al. Effects of ovariectomy on bone turnover, porosity, and biomechanical properties in ovine compact bone 12 months postsurgery. J Orthop Res 2009; 27: 303-9.

74 Ferretti M, Bertoni L, Cavani F, Zavatti M, Resca E, Carnevale G, et al. Influence of ferutinin on bone metabolism in ovariectomized rats. II: role in recovering osteoporosis. J Anat 2010; 217: 48-56.

75 Sharma V, McNeill JH. To scale or not to scale: the principles of dose extrapolation. Br J Pharmacol 2009; 157: 907-21.

76 Reagan-Shaw S, Nihal M, Ahmad N. Dose translation from animal to human studies revisited. FASEB J 2008; 22: 659-61.

77 GlaxoSmithKline. Wellbutein ${ }^{\circledR}$ Prescriping Information FDA 2004: $1-20$. 